\title{
Simple Heuristics and PTASs for Intersection Graphs in Wireless Ad Hoc Networks
}

\author{
Xiang-Yang Li \\ Dept. of Computer Science \\ Illinois Institute of Technology \\ 10, W. 31st Street \\ Chicago, IL 60616 \\ xli@cs.iit.edu
}

\author{
Yu Wang \\ Dept. of Computer Science \\ Illinois Institute of Technology \\ 10, W. 31st Street \\ Chicago, IL 60616 \\ wangyu1@iit.edu
}

\begin{abstract}
In wireless ad hoc networks, each wireless device has a transmission range, which is usually modeled as a disk centered at this node. A wireless node can send message directly to all nodes lying inside this disk. We present several intersection graphs to model the wireless networks. Then we present some simple heuristics and/or PTASs to approximate the maximum independent set, the minimum vertex cover and the minimum graph coloring in these graph models.
\end{abstract}

Categories and subject descriptors: C.2.1 Network Architecture and Design-Wireless communication; F.2.2 Nonnumerical Algorithms and Problems - Geometrical problems and computations; G.2.2 Graph Theory-Network problems.

\section{General Terms: Algorithms.}

keywords: Independent set, vertex cover, vertex coloring, disk graphs, PTAS, wireless ad hoc networks.

\section{INTRODUCTION}

Mobile wireless networking has received significant attention over the last few years due to its wide potential applications in various situations such as battlefield, emergency relief and so on. There are no wired infrastructures or cellular networks in wireless ad hoc networks. Multi-hop communication (carried out by the relaying of intermediate nodes) is required when the receiver node is not within the sender's transmission range. Thus, each wireless node also acts as a router, forwarding data packets for other nodes. Each wireless node often has an omni-directional antenna, i.e., a single transmission of a node can be received by any node within its vicinity. The transmission range of a node is often modeled as a disk centered at this node. When all wireless nodes have the same transmission radius, the wireless ad hoc networks are often modeled by the unit disk graph (UDG), in

Permission to make digital or hard copies of all or part of this work for personal or classroom use is granted without fee provided that copies are not made or distributed for pro£t or commercial advantage and that copies bear this notice and the full citation on the frst page. To copy otherwise, to republish, to post on servers or to redistribute to lists, requires prior specifc permission and/or a fee.

Dial-M'02, September 28, 2002, Atlanta, Georgia, USA.

Copyright 2002 ACM 1-58113-587-4/02/0009 ...\$5.00. which two nodes are connected iff their Euclidean distance is no more than the transmission radius. However, generally, different nodes will have different transmission radius due to various workloads and tasks performed. Thus, we apply the intersection graph and its variations to model the wireless networks.

Intersection graphs are graphs each of whose vertices is represented by a set, and two vertices are connected if their corresponding sets have a non-empty intersection. Intersection graphs have been widely studied $[4,11,12]$. The example of intersection graphs includes interval graph [9], unit disk graph $[1,2,10]$, and coin graph [14]. In this paper, we define several new intersection graph models to capture the communication properties of wireless networks. It is wellknown that many NP-hard problems remain NP-hard even they are restricted to intersection graph model. Moreover, the maximum independent set (MIS) problem, the minimum vertex cover (MVC) problem, and the minimum graph coloring (MGC) problem remain NP-hard even we restrict them to unit disk graph. In this paper we present some simple heuristics and PTASs to approximate these problems in the intersection graph models.

\subsection{Preliminaries}

We consider a wireless ad hoc network consisting of a set $V$ of wireless nodes distributed in a two-dimensional plane. In addition, we assume that the nodes are static or can be viewed as static during a reasonable period of time. Each node $v \in V$ has a transmission radius $r_{v}$. Let $D\left(v, r_{v}\right)$ denote the disk centered at $v$ with radius $r_{v}$. Then the set of wireless node $V$ defines a set of disks $\mathcal{D}=\left\{D\left(v, r_{v}\right) \mid v \in V\right\}$ in the two dimensional plane. Generally, without specifically expressing, we assume that the geometry location of a node $v$ is unknown, but the transmission radius $r_{v}$ is known by node $v$ and each node $v$ knows all its 1-hop neighbors in the graph models (proposed later). Let $\|u v\|$ denote the Euclidean distance of two wireless nodes $u$ and $v$.

A disk graph is the intersection graph of the set of disks defined by wireless nodes $V$. In other words, two nodes $u$ and $v$ are connected if $\|u v\| \leq r_{u}+r_{v}$. In wireless ad hoc networks, if two nodes $u$ and $v$ are not connected in the disk graph, then they can transmit messages simultaneously without causing any signal interferences. Therefore, the chromatic number of the disk graph is the upper-bound of the number of channels needed by all wireless nodes so that they can communicate without interferences. Here we 
assume that a wireless node can tune its receiving device to different channels other than its transmission channel. The disk graph model actually captures the primal interference among all wireless nodes.

However, the disk graph model is an over-estimation for interferences. For example, when two disks intersect, i.e., the transmission region of two corresponding wireless nodes overlap, these two nodes can still use the same channel if their common transmission region does not contain any other wireless node inside. Then we define the interference graph as follows: two nodes $u$ and $v$ are connected if there is a node inside their common transmission region, i.e., $D\left(u, r_{u}\right) \cap D\left(v, r_{v}\right)$ contains some node from $V$. The interference graph model takes not only the primal interferences but also the secondary interferences into account.

Remember that, for wireless ad hoc networks, when node $u$ is not within the transmission region of $v$, then $v$ cannot directly communicate with $u$; it needs some other intermediate nodes to relay the message. When a node $v$ sends a message to some node $w$ within its transmission range, several existing wireless protocols require a direct acknowledgment from node $w$. Then we can model the ack-based communication by the ack-communication graph. Two nodes $u$ and $v$ are connected if $\|u v\| \leq \min \left(r_{u}, r_{v}\right)$.

The last interesting model is the conflict graph, in which two nodes $u$ and $v$ are connected, i.e., conflicted, if $\|u v\| \leq$ $\max \left(r_{u}, r_{v}\right)$. Then the chromatic number of the conflict graph is a lower-bound of the number of channels needed by all wireless nodes so that they can communicate without interferences.

For a given set of wireless nodes $V$ with a function $R$ specifying the transmission radius of each node, we let $D G(V, R)$, $I G(V, R), A G(V, R)$, and $C G(V, R)$ denote the disk graph, the interference graph, the ack-communication graph, and the conflict graph defined by $V$ and $R$. When it is clear from the context, we will omit $R$ or even $V$. It is easy to show that $A G \subseteq C G \subseteq I G \subseteq D G$.

A subset of vertices in a graph $G$ is an independent set if for any pair of vertices, there is no edge between them. It is a maximal independent set if no more vertices can be added to it and it still be an independent set. It is a maximum independent set (MIS) if no other independent set has more vertices. The goal of the MIS problem is to compute, given a graph, a subset of pair-wisely unconnected vertices with maximum cardinality.

Given a graph $G=(V, E)$, a subset $V^{\prime} \subseteq V$ of vertices is a vertex cover if for every edge in $G, V^{\prime}$ contains at least one of its end-vertices. In other words, every edge is dominated by some node from $V^{\prime}$. The goal of minimum vertex cover (MVC) problem is to find a vertex cover with minimum cardinality. If the vertex of the graph is weighted, then the minimum weighted vertex cover problem is to find a vertex cover with minimum total weight.

The graph coloring (GC) problem (often called vertex coloring problem) is to assign each vertex a color so that two adjacent vertices have different colors. The minimum graph coloring (MGC) problem is to use minimum number of colors so a valid vertex coloring can be obtained.

In this paper, we are interested in designing efficient algorithms to approximate the maximum independent set, the minimum vertex cover, and the minimum graph coloring for the graph models defined above.

Given a graph $G$ and a problem $P$, let $O P T_{P}(G)$ de- note an optimum solution of problem $P$ when the input graph is $G$. An algorithm is a $\rho$-approximation algorithm for a problem $P$ if, given any input graph $G$, it runs in polynomial time and always computes a solution that is at least $\frac{1}{\rho} O P T_{P}(G)$ for maximization-based $P$ and at most $\rho \cdot O P T_{P}(G)$ for minimization-based $P$. An algorithm is a polynomial-time-approximation-scheme (PTAS) if, for any additional parameter $\varepsilon>0$, it always computes a solution that is at least $\frac{1}{1+\varepsilon} O P T_{P}(G)$ for maximization based problem $P$ and at most $(1+\varepsilon) \cdot O P T_{P}(G)$ for minimization based problem $P$.

\subsection{Previous Results}

For unit disk graphs, Marathe et al. [10] gave simple centralized heuristics to approximate the maximum independent set, the minimum vertex cover, the minimum vertex coloring, the minimum dominating set, and the minimum connected dominating set within constant $3, \frac{3}{2}, 3,5$, and 10 respectively. Hunt et al. [8] then presented the first PTAS to approximate the maximum independent set, the minimum vertex cover, and the minimum dominating set in UDG.

For disk graphs, it was claimed in [10] that the MIS problem can be approximated within 5. Then Erlebach [3] proposed an elegant PTAS for maximum weighted independent set (MWIS) and the minimum weighted vertex cover (MWVC) based on the shifting strategy used by Hochbaum [7] and Hunt et al. [8]. The algorithm runs in time $\frac{1}{\varepsilon^{2}} n^{O\left(\frac{1}{\varepsilon^{4}}\right)}$. Thus, when the wireless network is modeled by a disk graph, we already have PTASs for maximum independent set and minimum vertex cover problems.

\subsection{Our Results}

In this paper, we present PTASs or efficient algorithms to approximate MIS, MVC and MGC when the wireless networks are modeled by disk graphs, interference graphs, ackcommunication graphs, and conflict graphs. Some of our algorithms need only every node's transmission radius and the graph structure, while the PTASs need the exact geometry location of each node as extra. We present PTASs for MIS and MVC when the network is modeled by disk graphs, interference graphs, ack-communication graphs, and conflict graphs. We also show that the graph coloring problem in these graph models can be approximated with 5 for disk graphs, ack-communication graphs, and conflict graphs and 40 for interference graphs.

The rest of the paper is organized as follows. In Section 2, we show that the previous centralized method for computing MIS in disk graph model still works in other models introduced in this paper. However, this method does not extend naturally to minimum dominating set. We also give a PTAS for approximating the maximum weighted independent set. In Section 3, we present modified algorithms suitable for approximating the minimum weighted vertex cover. Section 4 is devoted to study the approximation of graph coloring. We conclude our paper in Section 5 by pointing out some possible future research directions.

For easy reading, we summarize the notations used in this paper in Table 1. In these notations, the definition of $i n$ dependence and non-independence are different in various graph models. Figure 1 shows the situations in which two disks $D_{v}$ and $D_{u}$ are not independent under different graph models. Similarly, it may have different meaning that a disk $D_{i}$ could intersect a geometry square $S$. In the disk graph 
model, the conflict graph model, and the interference graph model, a disk $D_{i}$ is said to intersect a geometry square $S$ if it intersects the square $S$ geometrically. However, in the ack-communication graph model, we apply a much stronger condition: a disk $D_{i}$ is said to intersect a geometry square $S$ if its center $v_{i}$ is inside $S$. The reason is that if $v_{i}$ is outside of $S$, then it is impossible for $v_{i}$ to connect to any node $v_{j}$ with the disk $D_{j}$ contained inside $S$. Therefore, for ack-communication graph model, we only consider the disks whose centers are inside $S$ instead of all the disks which geometrically intersect $S$ in our algorithms. We say a disk $D_{i}$ covers a disk $D_{j}$ if $v_{i} v_{j}$ is an edge in the corresponding graph model.

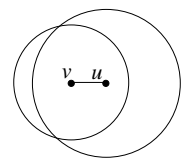

(a) ack-communication

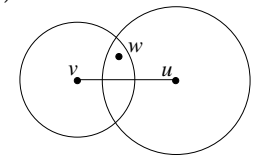

(c) interference graph

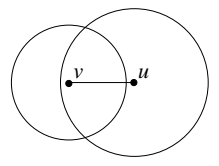

(b) conflict graph

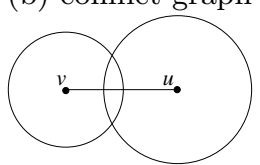

(d) disk graph
Figure 1: Non-independence in our graph models.

\section{MAXIMUM INDEPENDENT SET}

The problem of constructing MIS is well-known to be a NP-hard problem even restricted to UDG [2]. It is easy to show that UDG is a special case of all graph models introduced in this paper. Thus, constructing MIS remains NP-hard when restricted to the graphs defined in this paper. For disk graphs, Marathe et al. [10] presented an algorithm that is easy to implement and approximates the MIS within 5. Recently, Erlebach [3] proposed a PTAS for MIS on disk graph based on the shifting strategy $[7,8]$. In this section, we show that these methods work well in all graph models.

\subsection{Simple Approximation Method}

Assume that we know the transmission radius $r_{v}$ of each wireless node $v$. In addition, we also know the graph representation of the underlying graph. The algorithm (see [10]) first finds the node with the smallest transmission radius, and adds it to the independent set; then removes this node and all its neighbors from the graph. Repeat the above steps until the graph is empty. Obviously, this algorithm does compute a maximal independent set with $O(n \log n)$ running time. We then prove the following theorem that guarantees the quality of the computed independent set.

THEOREM 1. The computed IS has size at least $\frac{1}{5}$ of that of MIS if the input graph is a disk graph, an ack-communication graph, or a conflict graph.

Proof. We claim that, for any node $u \in I S$, there are at most 5 nodes from an optimum MIS solution such that they are connected to $u$ and are removed by the algorithm due to the removing of $u$. Notice that, every node from an optimum solution is connected to some node from IS computed by our algorithm because $I S$ is maximal. Then it immediately implies our theorem.

To prove our claim, we will show that if $v_{1}$ and $v_{2}$ from an optimum solution are connected to $u$ and removed by $u$, then $\angle v_{1} u v_{2}>\frac{\pi}{3}$. The selection of node $u$ always implies that $r_{u} \leq r_{v_{i}}$ for $i=1,2$. We prove this for different input graph models.

If the input graph is a disk graph, $u v_{i}$ is an edge implies that $\left\|u v_{i}\right\| \leq r_{u}+r_{v_{i}}$ for $i=1,2$. Notice that, here, nodes $v_{1}$ and $v_{2}$ cannot belong to $I S$. Because $v_{1}$ and $v_{2}$ are independent, $\left\|v_{1} v_{2}\right\|>r_{v_{1}}+r_{v_{2}}$. Thus, $\left\|v_{1} v_{2}\right\|>\left\|u v_{i}\right\|$ for $i=1,2$. In other words, $\angle v_{1} u v_{2}>\frac{\pi}{3}$.

If the graph is an ack-communication graph, $u v_{i}$ is an edge implies that $\left\|u v_{i}\right\| \leq \min \left(r_{u}, r_{v_{i}}\right)=r_{u}$ for $i=1,2$. Because $v_{1}$ and $v_{2}$ are independent, $\left\|v_{1} v_{2}\right\|>\min \left(r_{v_{1}}, r_{v_{2}}\right) \geq r_{u}$. Thus, $\left\|v_{1} v_{2}\right\|>\left\|u v_{i}\right\|$ for $i=1,2$. In other words, $\angle v_{1} u v_{2}>$ $\frac{\pi}{3}$.

If the graph is a conflict graph, $u v_{i}$ is an edge implies that $\left\|u v_{i}\right\| \leq \max \left(r_{u}, r_{v_{i}}\right)=r_{v_{i}}$ for $i=1,2$. Because $v_{1}$ and $v_{2}$ are independent, $\left\|v_{1} v_{2}\right\|>\max \left(r_{v_{1}}, r_{v_{2}}\right)$. Thus, $\left\|v_{1} v_{2}\right\|>$ $\left\|u v_{i}\right\|$ for $i=1,2$. In other words, $\angle v_{1} u v_{2}>\frac{\pi}{3}$.

Notice that the above proof cannot be directly applied to interference graphs. Unlike the proof of Theorem 1, we cannot show that, if $v_{1}$ and $v_{2}$ from an optimum solution are connected to $u$ and removed by $u$, then $\angle v_{1} u v_{2}>\frac{\pi}{3}$. Instead, we have

TheOrem 2. The computed IS has size at least $\frac{1}{40}$ of that of MIS if the input graph is an interference graph.

Proof. We prove this using an area argument. Consider any node $u$ selected by our algorithm. The nodes from an optimum solution, which are connected to $u$ and removed by $u$, can be partitioned into two cases: outside $D_{u}$ or inside $D_{u}$.

Let $v_{1}, v_{2}, \cdots, v_{k} \notin D_{u}$ be the $k$ nodes from the optimum solution that are connected to $u$ and are removed by the algorithm due to the removing of $u$. The selection of $u$ implies that $r_{v_{i}} \geq r_{u}$ for all $i=1,2, \cdots, k$. In addition, the disk $D_{i}$ centered at $v_{i}, i=1,2, \cdots, k$ cannot contain any node $v_{j}, j \neq i$ inside because all disks centered at $i=$ $1,2, \cdots, k$ are independent in the interference graph model. Nodes $v_{i}, i=1,2, \cdots, k$ is connected to $u$ implies that $D_{i}$ intersects with disk $D_{u}$. Let $B_{u}$ be the disk centered at $u$ with radius $2 r_{u}$ and $T_{u}=B_{u}-D_{u}$. Then every disk $D_{i}$, $i=1,2, \cdots, k$, will intersect $T_{u}$. It is not difficult to show that $T_{u} \cap D_{i}$ achieves the smallest area when $v_{i}$ is on the boundary of $B_{u}$ and $r_{v_{i}}=r_{u}$. See Figure 2. We can show that $\angle w v_{2} u>\frac{5}{12} \pi$. Thus, the smallest area is at least $\frac{5}{12} \pi$. Notice that the area of $T_{u}$ is $3 \pi$.

Notice that the region $T_{u} \cap D_{i}$ and $T_{u} \cap D_{j}$ for $1 \leq i, j \leq k$ may overlap. However, we will show that every point $x$ is covered by at most 5 disks from $D_{i}, i=1,2, \cdots, k$. See Figure 3. Assume node $x$ is covered by two disks $D_{i}$ and $D_{j}$, i.e., $\left\|x v_{i}\right\| \leq r_{v_{i}}$ and $\left\|x v_{j}\right\| \leq r_{v_{j}}$. Then $\left\|v_{i} v_{j}\right\|>$ $\max \left(r_{v_{i}}, r_{v_{j}}\right)$ because $D_{i}$ and $D_{j}$ are independent in the interference graph, which implies that $\angle v_{i} x v_{j}>\frac{\pi}{3}$. Thus, $x$ is covered by at most 5 independent disks. Therefore, by an area argument, we have $k \cdot \frac{5}{12} \pi<5 \cdot 3 \pi$. Thus, $k \leq 35$.

Let $v_{1}, v_{2}, \cdots, v_{h} \in D_{u}$ be the $h$ nodes from the optimum solution that are connected to $u$ and are removed by the algorithm due to the removing of $u$. Then obviously, all 


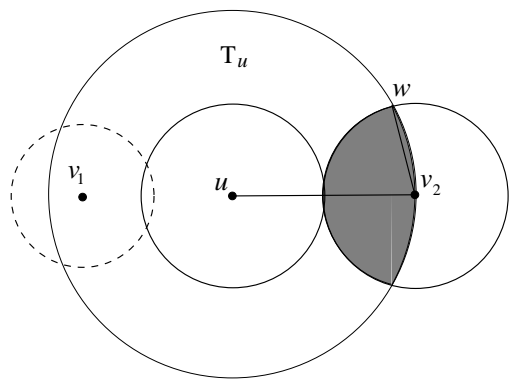

Figure 2: The number of independent neighbors removed by $u$ is bounded.

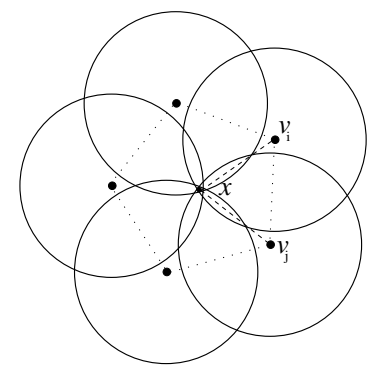

Figure 3: The number of independent disks which cover any point $x$ is bounded by 5 .

disks centered at $v_{i}, i=1,2, \cdots, h$ contain node $u$ but not any other node. Then similarly, we have $\angle v_{i} u v_{j}>\frac{\pi}{6}$ for $1 \leq i, j, \leq h$. Thus $h \leq 5$.

Consequently, there are at most $35+5=40$ independent nodes are removed when we remove all nodes adjacent to a node $u$ selected by our algorithm.

Figure 4 gives a configuration example such that there are 12 independent nodes that are removed. These 12 nodes $v_{i}$, $i=1,2, \cdots, 12$ are equally distributed on the circle centered at $u$ with radius $2 r_{u}$. The transmission radius of each of them is set as $r_{u}+\epsilon$ for a very small positive $\epsilon$. A node $w_{i}, i=1,2, \cdots, 12$, is placed on segment $u v_{i}$ and $\left\|u w_{i}\right\|=$ $r_{u}$. Thus, node $u$ has interference with all 12 nodes $v_{i}$, $i=1,2, \cdots, 12$, and these 12 nodes are independent with each other. It then implies that the lower bound on the

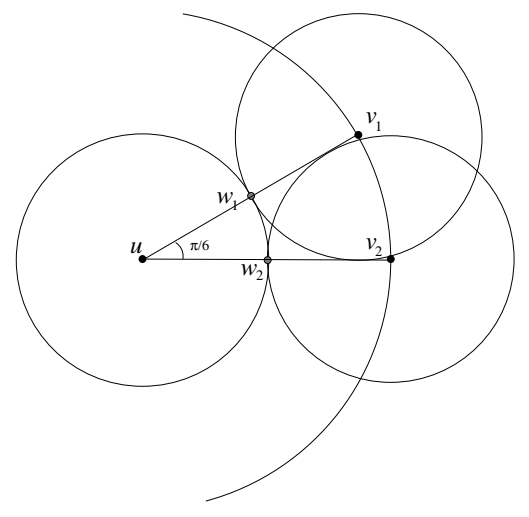

Figure 4: 12 independent neighbors removed by $u$.

approximation ratio for MIS of the smallest radius heuristic is at least 12 for interference graph model. We believe that 12 is also the tight upper bound, but to prove that, a more rigorous analysis is needed.

This simple approximation method for MIS can be easily converted to a distributed one that is suitable wireless ad hoc networks. It is omitted here due to space limit.

\subsection{PTAS for MWIS}

Assume that we are given a set $\mathcal{D}=\left\{D_{1}, D_{2}, \cdots, D_{n}\right\}$ of $n$ disks in the two-dimensional plane, where disk $D_{i}$ has transmission radius $r_{i}$, center $v_{i}=\left(x_{i}, y_{i}\right)$ and a weight $w\left(D_{i}\right)=w_{i}$. For a subset of disks $U \subseteq \mathcal{D}$, let $w(U)=$ $\sum_{D_{i} \in U} w\left(D_{i}\right)$, i.e., the summation of the weights of disks in $U$. Two disks $D_{i}$ and $D_{j}$ are said to be independent if the two nodes $v_{i}$ and $v_{j}$ are not connected in the corresponding graph model. Figure 1 shows the non-independence in different graph models.

The shifting strategy [8] was used to develop a PTAS for the unit disk graph. The plane is subdivided into grid of size at $m$ by $m$ for some integer $m$ by a collection of vertical lines $x=i \cdot m$ and horizontal lines $y=j \cdot m$. A subdivision is called $(r, s)$-shifting if it is formed by a collection of vertical lines $x=i \cdot m+r$ and horizontal lines $y=j \cdot m+s$. A square is formed by two consecutive vertical lines and two consecutive horizontal lines in a $(r, s)$-shifting. For each square, an optimal solution of MIS is obtained in polynomial time for all disks contained in the square but not intersecting the boundary of the square. The union of the MIS in all squares is returned as the final solution for this shifting. It is not difficult to show that the result is an independent set. The pigeonhole principal implies that there is a shifting of the subdivision such that the size of the computed independent set is at least $\left(1-\frac{1}{m}\right)^{2}$ of optimum.

The basic approach of the PTAS for approximating MIS in various graph models introduced in this paper is similar to that of [3], i.e., to divide the transmission disks into different level according to its radius. At same level, all nodes have similar transmission radii. As in [3], we scale all disks so that the largest disk has transmission radius $\frac{1}{2}$. Let $r_{\text {min }}$ be the smallest transmission radius among all wireless nodes. Let $k>1$ be a fixed integer and $\ell=\left\lfloor\log _{k+1} \frac{1}{2 r_{\text {min }}}\right\rfloor$. We partition $\mathcal{D}$ into $\ell+1$ levels such that level $j, 0 \leq j \leq \ell$, consists of all disks $D_{i}$ with transmission radius satisfying

$$
\frac{1}{(k+1)^{j+1}}<2 r_{i} \leq \frac{1}{(k+1)^{j}} .
$$

Let $l\left(D_{i}\right)$ denote the level of disk $D_{i}$, i.e., $l\left(D_{i}\right)=\left\lfloor\log _{k+1} \frac{1}{2 r_{i}}\right\rfloor$.

As did for UDG, for each level $j$, we subdivide the plane into grid by using a set of vertical lines $L_{j, v}: x=v \frac{1}{(k+1)^{j}}, v \in$ $Z$ and a set of horizontal lines $H_{j, h}: y=h \frac{1}{(k+1)^{j}}, h \in Z$. Hereafter $j$ is called the level of the lines; $v$ (and $h$ ) is called the index of the vertical (and horizontal) line $l_{j, v}$ (and $h_{j, h}$ ) at level $j$. A $(r, s)$-shifting of the subdivision is the grid defined by the set of vertical lines whose indices modulo $k$ equal $r$ and the set of horizontal lines whose indices modulo $k$ equal $s$. It was proved in [3] that a vertical line at level $j$ of a $(r, s)$-shifting subdivision is also a vertical line at level $j+1$ of the $(r, s)$-shifting subdivision.

Any two consecutive vertical lines at level $j$ whose indices modulo $k$ equal $r$, and any two consecutive horizontal lines at level $j$ whose indices modulo $k$ equal $s$, form a $j$-square in the $(r, s)$-shifting subdivision. See Figure 5 for an illus- 
tration of a 0 -square for $r=s=0$ and $k=3$. Here the solid lines are all lines at level 0 and all dashed lines are all lines at level 1 . The $j$-squares are represented by thicker lines. It is easy to show that any $j$-square $S$ is subdivided into $(k+1)^{2}(j+1)$-squares (by lines $L_{j+1, v}$ and $H_{j+1, h}$ at level $j+1)$. Notice that it only contains $k^{2}$ grids defined by lines at level $j$. These $(j+1)$-squares $S^{\prime}$ are called the children of $S$, denoted by $S^{\prime} \prec S$. And $S$ is called the parent of $S^{\prime}$. It is easy to show that any $j$-square $S$ has length $\frac{k}{(k+1)^{j}}$. Thus it can contain some disks inside with level at least $j-1$, but not any disks inside with level less than $j-1$.

A disk $D_{i}$ with center $\left(x_{i}, y_{i}\right)$ and radius $r_{i}$ is said to hit a vertical line at $x=a$ if $a-r_{i}<x_{i} \leq a+r_{i}$. Similarly, we say the disk $D_{i}$ hits a horizontal line at $y=b$ if $b-r_{i}<$ $y_{i} \leq b+r_{i}$. In other words, a disk hits a line if it intersects this line, except that it only touches the line from the left or from the bottom. For maximum independent problem, a disk $D_{i}$ at level $l\left(D_{i}\right)=j$ is said to be active (respecting to shifting $(r, s))$ if it does not intersect the boundary of any $j$-square of the $(r, s)$-shifting subdivision. The definition of active disks for minimum vertex cover is different, which will be discussed later. For a $j$-square $S$, let $\mathcal{D}_{S}^{<j}$ be the set of active disks with level less than $j$ and intersect with $S$. Similarly, we define $\mathcal{D}_{S}^{\leq j}, \mathcal{D}_{S}^{=j}, \mathcal{D}_{S}^{>j}$, and $\mathcal{D}_{S}^{\geq j}$. For a $j$-square $S$, let $O P T_{S}^{<j}$ be the set of disks from $O P T\left(\mathcal{D}_{S}\right)$ with level less than $j$ and intersect with $S$. Similarly, we define $O P T_{S}^{\leq j}, O P T_{S}^{=j}, O P T_{S}^{>j}$, and $O P T_{S}^{\geq j}$.

For each level $j$, let $\mathcal{D}_{j}(r, s)$ be the set of active disks at level $j$ respecting to shifting $(r, s)$. Define $\mathcal{D}(r, s)=$ $\cup_{j=0}^{\ell} \mathcal{D}_{j}(r, s)$, i.e., the union of active disks at all levels respecting to shifting $(r, s)$. Then a $j$-square $S$ is called relevant if $\mathcal{D}(r, s)$ contains at least one disk of level $j$ that is inside $S$ in the corresponding graph model. More rigorous definition of relevant will be given later, which depends on the graph models. Let $O P T_{I S}\left(\mathcal{D}^{\prime}, G\right)$ denote the weight of the maximum weighted independent set for a set of disks $\mathcal{D}^{\prime}$ when the network is modeled by graph model $G$. We will omit $G$ and/or $I S$ when it is clear from the context. The following proof is given by Erlebach [3]. We found that the correctness of this lemma does not depend on the graph model. We include it here for the completeness of presentation.

Lemma 3. There is at least one shifting $(r, s), 0 \leq r, s<$ $k$ such that

$$
\operatorname{OPT}(\mathcal{D}(r, s)) \geq\left(1-\frac{1}{k}\right)^{2} \cdot \operatorname{OPT}(\mathcal{D})
$$

when the graph is modeled by the disk graph, the interference graph, the ack-communication graph, and the conflict graph.

Proof. Consider a maximum weighted independent set $S^{\star} \subseteq \mathcal{D}$ for any graph model introduced here. Let $S_{r}^{\star}$ be the set of disks $D_{i} \in S^{\star}$ such that the disk $D_{i}$ hits some vertical line $L_{j, v}$ at the level $j=l\left(D_{i}\right)$ whose index $v$ modulo $k$ equals $r$. Then $\cup_{r=0}^{k-1} S_{r}^{\star} \subseteq S^{\star}$. In addition, $S_{r}^{\star}, 0 \leq r \leq k-1$ are pair-wise disjoint. Thus,

$$
\sum_{r=0}^{k-1} w\left(S_{r}^{\star}\right) \leq w\left(S^{\star}\right)
$$

The pigeonhole principal implies that there is an index $r_{0}$ such that $w\left(S_{r_{0}}^{\star}\right) \leq \frac{1}{k} \cdot w\left(S^{\star}\right)$. Let $S_{\frac{r_{0}}{\star}}=S^{\star}-S_{r_{0}}^{\star}$. Therefore, $w\left(S_{\overline{r_{0}}}^{\star}\right) \geq\left(1-\frac{1}{k}\right) \cdot w\left(S^{\star}\right)$.
Using the same technique, we can show that there is an index $s_{0}$ such that the set of disks from $S_{\overline{r_{0}}}^{\star}$, each $D_{i}$ of which does not hit a horizontal line $H_{j, h}$ at its level $j=$ $l\left(D_{i}\right)$ and the index $h$ modulo $k$ equals $s_{0}$, has total weight at least $\left(1-\frac{1}{k}\right) \cdot w\left(S_{\overline{r_{0}}}^{\star}\right)$. Use $S_{\overline{r_{0}}}^{\star}, \overline{s_{0}}$ to denote such set of disks. Obviously, $S_{\overline{r_{0}}}^{\star}, \overline{s_{0}}$ is an independent set for $\mathcal{D}\left(r_{0}, s_{0}\right)$. Thus, there is a $\left(r_{0}, s_{0}\right)$-shifting such that the weight of the maximum weighted independent set in $\mathcal{D}\left(r_{0}, s_{0}\right)$ is at least $\left(1-\frac{1}{k}\right)^{2}$ of the optimum $O P T(\mathcal{D})$.

Before we show the PTASs for maximum weighted independent set for graph models introduced in this paper, we first exam the structural properties of an optimum solution for all disks in $\mathcal{D}(r, s)$ for $0 \leq r, s \leq k-1$. Given a graph model, an optimum solution cannot contain any disk that hits a line at level 0 of the $(r, s)$-shifting subdivision. In other words, each disk of the optimum solution $\operatorname{OPT}(\mathcal{D}(r, s))$ is contained inside some 0-square. Moreover, the optimum solution can be divided into two subsets. One contains some independent disks at level 0 , denoted by $I_{0}$. The other one contains independent disks at lower level that are independent with any disk from $I_{0}$. By the definition of $\mathcal{D}(s, r)$, all disks in the second subset cannot intersect any lines, with level 1, of the $(r, s)$-shifting subdivision. In other words, each disk in the second subset is contained inside some 1square. Figure 5 gives an example of optimum solution in a 0 -square. Here $k=3$ and $r=s=0$. The disks with the thickest boundary are at level 0 . The disks that are not active is represented by dashed boundary. The shaded disks are in the optimum solution.

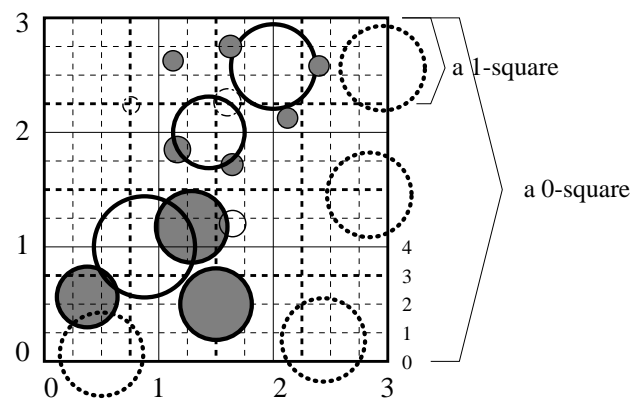

Figure 5: An optimum solution for a 0-square.

The above partition of disks in the optimum solution in a 0 -square can be performed recursively down to the squares at level $\ell$ as follows. Given a $j$-square $S$, let $I$ be a set of independent disks of level smaller than $j$, each of which intersects $S$. Let $M W I S(S, I)$ be a maximum weighted independent set of disks that are contained in $S$ (must be of level at least $j$ ) and independent from the disks in $I$. Then the union of $M W I S(S, \emptyset)$ for all relevant squares $S$ without parent must be the optimum solution for $\mathcal{D}(r, s)$.

We then discuss in detail how to compute $\operatorname{MWIS}(S, I)$ using dynamic programming. Assume that we already compute the entry $M W I S(S, I)$ for all squares $S$ with level at least $j+1$ and all appropriate independent set $I$ intersecting $S$. We show how to compute $\operatorname{MWIS}(S, I)$ for a $j$-square $S$ and some independent set of disks $I$ intersecting $S$. The disks in $M W I S(S, I)$ can be divided into two subsets. One, denoted by $X$, contains some independent disks inside $S$ with level $j$ that are independent with disks from $I$. The other one contains independent disks at level larger than $j$ 
that are independent with any disk from $I$ and $X$. By the definition of $\mathcal{D}(s, r)$, all disks in the second subset cannot intersect any lines, with level $j+1$, of the $(r, s)$-shifting subdivision. In other words, each disk in the second subset is contained inside some $(j+1)$-square $S^{\prime}$, which is contained in $S$. Thus, by properly choosing the set of disks $X$ (disks inside $S$ with level $j$ and independent with $I$ ), we can generate $M W I S(S, I)$ by

$$
M W I S(S, I)=\max _{X}\left(\left(\bigcup_{S^{\prime} \prec S} M W I S\left(S^{\prime}, I_{S^{\prime}} \cup X_{S^{\prime}}\right)\right) \cup X\right) .
$$

Here $I_{S^{\prime}}$ is the subset of disks from $I$ that intersect $S^{\prime} . X_{S^{\prime}}$ is defined similarly.

It is not difficult to prove the correctness of the above dynamic programming approach. To guarantee that the above dynamic programming approach runs in polynomial time of the number of disks $n$ and $k$, we have to show that the size of $I_{S} \cup X_{S}$, i.e., the number of independent disks with level at most $j$ and intersecting a $j$-square $S$, is always bounded by a constant under the graph models introduced in this paper.

Lemma 4 (Bounded Independent Disks). Let $S$ be any $j$-square and let $I$ be a set of independent disks with level at most $j$, each of which may connect to some disk contained in $S$. Then there is a constant $C$ depending on the graph model and $k$ such that the cardinality of $I$ is at most $C$.

ProOF. We prove this lemma individually for each graph model introduced in this paper.

For disk graph model, Erlebach et al. [3] gave a constant $C=\frac{4}{\pi}(k+2)^{2}(k+1)^{2}$ using an area argument.

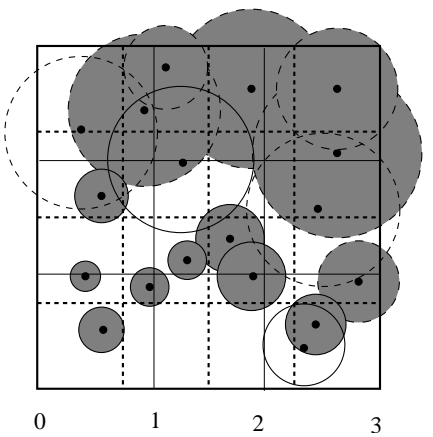

Figure 6: There are constant number of independent disks of level at most $j$, intersecting a $j$-square $S$ in the ack-communication graph model.

For ack-communication graph model, the disk whose center is outside of $S$ cannot have edge with any disk contained inside $S$. So if we add them to $M W I S(S, I)$, the resulted set is still guaranteed to be an independent set. Thus, unlike the disk-graph model, to bound $|I|$, we only have to consider the disks whose centers are inside $S$, i.e., the disks that can possibly "connect" with some disk contained inside $S$. Notice that all disks in $I$ has level at most $j$, which implies that all disks in $I$ has diameter at least $\frac{1}{(k+1)^{j+1}}$. Then the distance of the centers of any two disks $D_{p}$ and $D_{q}$ from $I$ is at least $\frac{1}{2(k+1)^{j+1}}$; otherwise, these two disks $D_{p}$ and
$D_{q}$ will not be independent. The $j$-square $S$ has side length $\frac{k}{(k+1)^{j}}$. Therefore, there are at most $C_{A G}$ independent disks whose centers are inside $S$. See Figure 6 . Here, by an area argument,

$$
C_{A G} \leq\left(\frac{k}{(k+1)^{j}}\right)^{2} /\left(\pi\left(\frac{1}{4(k+1)^{j+1}}\right)^{2}\right)=\frac{16 k^{2}(k+1)^{2}}{\pi} .
$$

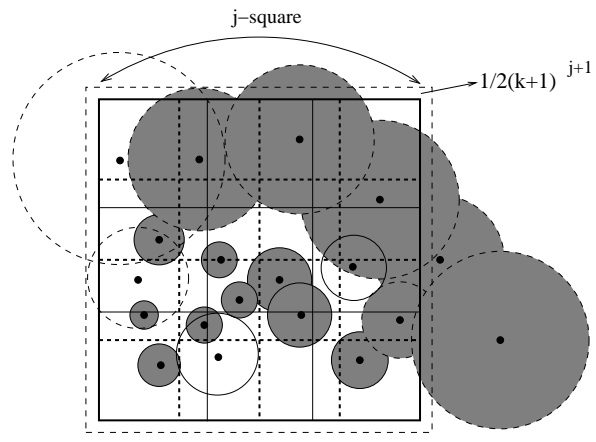

Figure 7: There are constant number of independent disks of level at most $j$ and intersecting a $j$-square $S$ in the conflict graph model.

For conflict graph model, again, we only have to consider all independent disks that could connect to some disks contained in $S$. Remember that, here, two disks $D_{p}$ and $D_{q}$ are independent if $v_{p}$ is not inside $D_{q}$ and $v_{q}$ is not inside $D_{p}$. Thus, the distance between the centers of any two disks from $I$ is at least $\frac{1}{2(k+1)^{j+1}}$. Using the same area argument, we can show that there are at most $\frac{16 k^{2}(k+1)^{2}}{\pi}$ independent disks with centers inside $S$ in the conflict graph model. Then we concentrate on estimating how many independent disks, denoted by $I_{O}$, such that (1) their centers are not inside $S$, (2) with level at most $j$, (3) each of them intersects $S$. We show that there are only a constant number of such disks by an area argument. Consider the four strips, denoted by $B(S)$, surrounded $S$ with width $\frac{1}{2(k+1)^{j+1}}$. See Figure 7 for an illustration. For a disk $D_{i} \in I_{O}$, it is not difficult to show that $B(S) \cap D_{i}$ achieves the smallest area when $v_{i}$ is on the boundary of $B(S)$ and $r_{v_{i}}=\frac{1}{2(k+1)^{j+1}}$. The smallest area of $B(S) \cap D_{i}$ is $\pi \frac{1}{8(k+1)^{2(j+1)}}$. Similar to Theorem 2, every point in $B(S)$ is covered by at most 5 disks from $I_{O}$. The area of $B(S)$ is $\frac{2 k(k+1)+1}{(k+1)^{2(j+1)}}$. Thus, the size of $I_{O}$ is at most

$$
5 \cdot \frac{2 k(k+1)+1}{(k+1)^{2(j+1)}} /\left(\pi \frac{1}{8(k+1)^{2(j+1)}}\right)=\frac{80 k(k+1)+40}{\pi}
$$

Thus, the total number of independent disks $I$ with level at most $j$ intersecting $S$ is at most

$$
C_{C G} \leq \frac{16 k^{2}(k+1)^{2}}{\pi}+\frac{80 k(k+1)+40}{\pi}
$$

For interference graphs, as we already showed that $C G \subseteq$ $I G$, any independent set in $I G$ is also an independent set in $C G$. We just proved that, for conflict graph model, the number of independent disks with level at most $j$ intersecting $S$ is bounded from above by constant $C_{C G}$. Thus, $C_{C G}$ is also an upper-bound of the number of independent disks with level at most $j$ intersecting $S$ for interference graph model. 
Remark: The number of independent disks that intersect a $j$-square in the ack-communication graph model is not bounded. It is easy to show that the disks $D_{i}, i \geq 1$, with center $v_{i}=\left(2^{i-1}(1+\epsilon)^{i}, 0\right)$ and radius $r_{i}=2^{i-1}(1+\epsilon)^{i}$ are independent; all such disks intersect any square containing the point $(0,0)$. Here $\epsilon$ is a small positive real number.

The algorithm processes all relevant squares in order of non-increasing levels. For each $j$-square $S$ and some appropriate independent set $I, M W I S(S, I)$ is computed by dynamic programming as follows.

\section{Algorithm 1. Compute $M W I S(S, *)$}

Let $R$ be all disks in $\mathcal{D}(r, s)$ of level at most $j$ intersecting $S$. For ack-communication graph model, we only consider disks of level at most $j$ and with centers inside $S$.

For all $J \subseteq R$ with at most $C$ disks \{

If $J$ is an independent set then

Let $X$ be all disks in $J$ with level $j$.

For each child square $S^{\prime}$ of $S\{$

Let $I^{\prime}$ be disks in $J$ intersecting $S^{\prime}$.

$X=X \cup M W I S\left(S^{\prime}, I^{\prime}\right)$. $\}$

Let $I$ be disks in $J$ with level less than $j$.

If $w(X)>w(M W I S(S, I))$, then $M W I S(S, I)=X$.

For the base situation, we can try all the possible independent sets to get the optimum solution in the largest level $\ell$. The output of the algorithm is the union of the $M W I S(S, \emptyset)$, taken over all relevant squares $S$ that do not have a parent. As did in [3], we can easily prove that the running time of this algorithm is $O\left(k^{2} n^{C}\right)$. Here $C$ is the constant in Lemma 4. In summary, we obtain a PTAS for the MWIS in all various intersection graphs introduced in this paper.

\section{VERTEX COVER}

It is well-known that the minimum weighted vertex cover for a general graph can be approximated within 2 . In this section, we present a PTAS for the minimum weighted vertex cover problem for all graph models introduced here. As we did for approximating MWIS, we use the same partition of disks into levels and the same subdivision of the plane into squares at each level. In addition, all integer values $r$ and $s$ within interval $[0, k-1]$ are considered. We first study the structural properties of the MWVC. Let $O P T$ be the minimum weighted vertex cover for $\mathcal{D}$. For any pair of $r$ and $s$, Let $O P T V(r)$ be the set of disks $D_{i} \in O P T$ such that the disk $D_{i}$ hits some vertical line $L_{j, v}$ at the level $j=l\left(D_{i}\right)$ whose index $v$ modulo $k$ equals $r$; Let $O P T H(s)$ be the set of disks $D_{i} \in O P T$ such that the disk $D_{i}$ hits some horizontal line $H_{j, h}$ at the level $j=l\left(D_{i}\right)$ whose index $h$ modulo $k$ equals $s$. let $\operatorname{OPT}(r, s)=O P T V(r) \cup \operatorname{OPTH}(s)$.

LEMMA 5. There is a $\left(r_{0}, s_{0}\right)$-shifting such that the total weight of disks in OPT $\left(r_{0}, s_{0}\right)$ is no more than $\frac{2}{k}$ of the weight of $O P T$.

Proof. Obviously, OPTV $(r)$ are pairwise disjoint for $0 \leq$ $r<k$ and $\sum_{r=0}^{k-1} w(O P T V(r))=w(O P T)$. Pigeonhole principal implies that there is $r_{0}$ such that $w\left(O P T V\left(r_{0}\right)\right) \leq$ $\frac{1}{k} w(O P T)$. Similarly, there exists $s_{0}$ such that $w\left(O P T H\left(s_{0}\right)\right) \leq$ $\frac{1}{k} w(O P T)$. Therefore, $w\left(O P T\left(r_{0}, s_{0}\right)\right) \leq w\left(O P T V\left(r_{0}\right)\right)+$ $w\left(O P T H\left(s_{0}\right)\right) \leq \frac{2}{k} w(O P T)$.
Notice that the correctness of Lemma 5 does not depend on the graph model.

For independent set problem, to guarantee that the union of an independent set in each child of a square $S$ is still an independent set, we only consider the independent disks that are totally contained in the children of $S$. In other words, a disk with level $j$ is active for independent set if it is geometrically inside some $j$-square. Contrary to MWIS, a vertex cover of disks inside a square could use some disks are not totally inside in this square. Then we have to define active disks for a $j$-square differently. As the vertex cover has to cover all edges, we associate each edge to at least one square and then compute the vertex cover for all edges associated with that $j$-square.

For the disk graph model, the interference graph model, and the conflict graph model, we associate an edge $u v$ to a $j$-square $S$ if $S$ intersects with $D_{u} \cap D_{v}$. Obviously, in any graph model introduced in this paper, if $u v$ is an edge then $D_{u}$ intersects $D_{v}$. In addition, there is always some square $S$ that has non-empty intersection with $D_{u} \cap D_{v}$. Thus, any edge $u v$ is associated with some square. See the left figure in Figure 8.

For the ack-communication graph model, we associate the edge $u v$ to a $j$-square $S$ if $S$ intersects with $D_{u} \cap D_{v}$ and $S$ contains $u$ inside and either (1) $r_{u}<r_{v}$ or (2) $r_{u}=r_{v}$ and the identity $I D(u)$ of $u$ is less than $I D(v)$. For any edge $u v$, assume that $u \in S_{1}$ and $r_{u} \leq r_{v}$. Then $S_{1} \cap\left(D_{u} \cap D_{v}\right) \neq \emptyset$ because $u \in D_{u} \cap D_{v}$. So edge $u v$ must be associated with square $S_{1}$. See the right figure in Figure 8 .

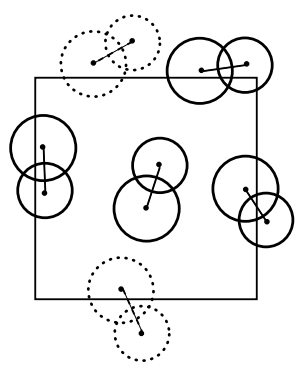

a) non-ack-communication

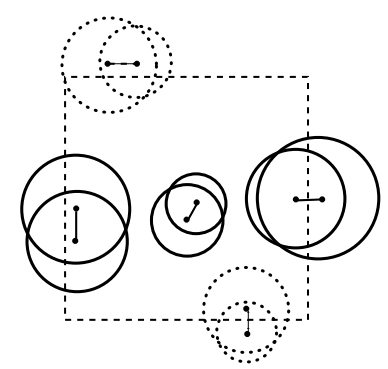

(b) ack-communication
Figure 8: Examples for the associated edges and the active disks in different graph models.

For a $j$-square $S$, a disk $D_{u}$ is said to be active for $S$ if there is an edge $u v$ or $v u$ associated with $S$ for some $v$. Thus, for edges associated with a $j$-square $S$, their vertex cover must be a subset of the active disks in $S$. Notice that an active disk $D_{u}$ for a $j$-square $S$ always intersects $S$. See Figure 8 as an illustration. Here the dashed disks are inactive, the solid disks are active for $S$. For all graph models introduced here, each disk is at most active for four $j$-squares on the same level of this disk.

A $j$-square $S$ is called relevant if there are some edges in the corresponding graph model associated with $S$. Given a $j$-square $S$, the presented algorithm will then construct a vertex cover for the edges associated with $S$ using disks active for $S$ knowing that some active disks $P$ are already chosen to be in the vertex cover. More precisely, given some subset $P$ of active disks for a $j$-square $S$, we will compute the minimum weighted set of active disks $X$, denoted by $V C(S, P)$, such that $P \cup X$ is a vertex cover for edges associated with $S$. 
For each $j$-square $S$, we consider all the disks each of which is incident on some edges associated with $S$. We then consider the optimum solution $O P T$ restricted to a square $S$, denoted by $O P T_{S}$, i.e., the disks in $O P T$ that are active for $S$. Then, $O P T_{S}$ is a vertex cover for all edges associated with $S$. Define active edges of $S$ as the set of edges $u v$ with $D_{u}$ and $D_{v}$ are active for $S$. Then the active edges of $S$ contains the associated edges of $S$. Thus, $O P T_{S}$ is also a vertex cover for all active edges of $S$.

Consider a relevant 0 -square $S$, the optimum solution $O P T_{S}$ can be divided into two subsets. One contains the disks which is a complement of an independent set of active disks for $S$ at level 0 , denoted by $P_{0}$. The other one contains disks at lower level that, together with $P_{0}$, can form a vertex cover for all edges active for $S$. Figure 9 gives an example of optimum solution restricted in a 0-square. Here $k=3$ and $r=s=0$. The disks with the thickest boundary are at level 0 . The disks that are not active is represented by dashed boundary. The un-shaded disks are in the optimum solution. The shaded disks are an independent set.

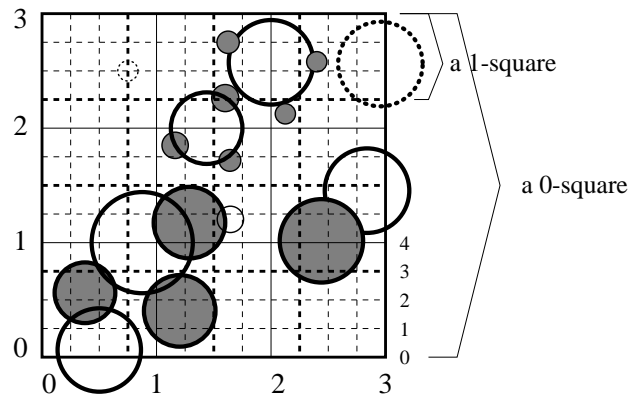

Figure 9: Active disks and a vertex cover for a 0square in disk graph model.

The above partition of disks in the optimum solution in a relevant 0-square can be performed recursively down to the squares at level $\ell$ as follows. Given a $j$-square $S$, let $R$ be the set of all active disks for $S$ with level at most $j$. Then any active edge using only disks from $R$ must have vertex cover from $R$. Thus for any vertex cover $U, R-U$ must be an independent set. Hereafter, we will use $J$ to denote a set of independent and active disks with level at most $j$ for a $j$-square $S$. Let $Q=R-J$ be the complement set of disks of $J$. Then, $Q$ can cover all disk in $R$. Let $P=Q^{<j}$. Let $V C(S, P)$ be the set of minimum weighted disks such that $V C(S, P) \cup P$ is a vertex cover for all active edges for $S$. Notice that, for optimum solution $O P T$ restricted to $S$, we can set $Q=O P T_{S}^{\leq j}, P=O P T_{S}^{<j}, J=R-O P T_{S}^{\leq j}$. The union of $V C(S, \emptyset)$ for all relevant squares $S$ without parent must be a vertex cover for the graph defined on $\mathcal{D}$.

A dynamic programming to compute $V C(S, \emptyset)$ is straightforward. The algorithm presented here is similar to that of [3]. It processes all relevant squares in order of nonincreasing levels. For each $j$-square $S$ and some set $P$, $V C(S, P)$ is computed as follows.

\section{Algorithm 2. Compute $\operatorname{VC}(S, P)$}

Let $R$ be all active disks for $S$ with level $\leq j$.

For all $J \subseteq R$ with at most $C_{2}$ disks \{

If $J$ is an independent set then

Let $Q=R-J$.
Let $X$ be all disks in $Q$ with level $j$.

For each relevant child square $S^{\prime}$ of $S\{$

Let $P^{\prime}$ be disks in $Q$ that is active for $S^{\prime}$.

$X=X \cup V C\left(S^{\prime}, P^{\prime}\right)$.

Let $P$ be disks in $Q$ with level less than $j$.

If $w(X)>w(V C(S, P))$, then $V C(S, P)=X$.

For the base situation (without relevant child), we actually try all the complement sets of possible independent active disks to get the optimum solution with minimum weight. The output of the algorithm is the union of the $V C(S, \emptyset)$, taken over all relevant squares $S$ that do not have a parent. Similar to Algorithm 1, the running time of this algorithm is $O\left(k^{2} n^{C_{2}}\right)$. Here $C_{2}$ is a constant that will be proved by the following Lemma 6 .

Lemma 6 (Bounded Independent Active Disks). Let $S$ be any $j$-square and let $J$ be a set of independent disks with level at most $j$ that is active for $S$. Then there is a constant $C_{2}$ depending on the graph model and $k$ such that the cardinality of $J$ is at most $C_{2}$.

Proof. For the disk graph models, the interference graph models, and the conflict graph models, all active disks intersect $S$. Then, the number of disks in $J$ is bounded by a constant using an area argument, which is the same as Lemma 4.

We then consider the ack-communication graph model. For each disk $D_{u} \in J$ with center $u$ outside $S$, there is a disk $D_{v}$ with level at least $j$ and $u v$ is an edge. For ackcommunication model, we know that $u$ must be inside $D_{v}$. Thus, $u$ is always inside the extended square $S^{\prime}$. Similar to Lemma 4, the number of independent active disks is at most

$$
C_{2}^{A G} \leq\left(\frac{1}{(k+1)^{j-1}}\right)^{2} /\left(\pi\left(\frac{1}{4(k+1)^{j+1}}\right)^{2}\right)=\frac{16(k+1)^{4}}{\pi} .
$$

This finishes the proof.

We then show that, given $S$ and $P$, our algorithm does compute $X=V C(S, P)$ such that $X \cup P$ is a vertex cover for all edges associated with $S$. For any edge $u v$ associated to $S$, without loss of generality, we assume that $r_{u} \leq r_{v}$.

If $D_{u}$ has level $j$, then both $D_{u}$ and $D_{v}$ is in $R$. Obviously, not both of $D_{u}$ and $D_{v}$ can belong to some independent set $J$. In other words, either $D_{u}$ or $D_{v}$ or both is selected to $Q$. If $D_{u}$ is selected to $Q$. Then $D_{u}$ is also selected to $X$. So $X \cup P$ covers edge $u v$. If $D_{u}$ is not selected to $Q$. Then $D_{v}$ is selected to $Q$. Then $D_{v}$ will be put into $X$ if $l\left(D_{v}\right)=j$ or it will be put to $P$ if $l\left(D_{v}\right)<j$. Thus, $X \cup P$ covers edge uv.

If $D_{u}$ has level larger than $j$, then edge $u v$ is also associated with some child $S^{\prime}$ of $S$. By induction, $V C\left(S^{\prime}, P^{\prime}\right)$ covers edge $u v$. From $V C(S, P)=X \cup \bigcup_{S^{\prime} \prec S} V C\left(S^{\prime}, P^{\prime}\right)$, we know that $u v$ is also covered by $V C(S, P)$.

Notice that every edge is active for some square. So the output of our algorithm is a vertex cover of $\mathcal{D}$. We then study the quality of the computed vertex cover. Let $O P T$ be the minimum weighted vertex cover of $\mathcal{D}$ in a graph model introduced in this paper. The following theorem proved that the shifting strategy does work for MWVC problem. Same theory was proved in [3] for disk graph model. 
THEOREM 7. For all graph models introduced here, there is a $(r, s)$-shifting, $0 \leq r, s<k$ such that for the vertex cover A generated by the algorithm,

$$
w(A) \leq\left(1+\frac{6}{k}\right) w(O P T) .
$$

Proof. Lemma 5 implies that there is a $\left(r_{0}, s_{0}\right)$-shifting such that $w\left(O P T\left(r_{0}, s_{0}\right)\right) \leq \frac{2}{k} w(O P T)$. We then consider the subdivision of the plane that results from this choice of $r_{0}$ and $s_{0}$. Let $\mathcal{R}$ be the set of all relevant squares, and $\mathcal{R}_{0}$ be the set of all relevant squares without parent. For any $j$-square $S$, let $O P T_{S}^{=j}$ denote the disks with level $j$ in $O P T$ that are active for $S$. Notice that $O P T=\bigcup_{S \in \mathcal{R}} O P T_{S}^{=j}$. Any disk can be active for at most 4 squares on its level. In addition, if a disk is active for some square $S$, it must intersect $S$. Thus, when we summarize $w\left(O P T_{S}^{=j}\right)$ over all relevant squares $S \in \mathcal{R}$, only disks in $O P T\left(r_{0}, s_{0}\right)$ are counted multiple times, whose weight $w\left(O P T\left(r_{0}, s_{0}\right)\right) \leq \frac{2}{k} w(O P T)$. Since each such disk is counted at most four times, and it must be counted once in $O P T$, we have

$$
\sum_{S \in \mathcal{R}} w\left(O P T_{S}^{=j}\right) \leq w(O P T)+3 \frac{2}{k} w(O P T)=\left(1+\frac{6}{k}\right) w(O P T) .
$$

Then, we only need to prove that the total weight of the output $A=\bigcup_{S \in \mathcal{R}_{0}} V C(S, \emptyset)$ is at most $\sum_{S \in \mathcal{R}} w\left(O P T_{S}^{=j}\right)$.

We already showed that the optimum solution $O P T$ restricted to $S$, denoted by $O P T_{S}$, is also considered by the algorithm because in one iteration we have $Q=O P T_{S}^{\leq j}$ and then $P=O P T_{S}^{<j}$. The algorithm computes the minimum weighted $X$ such that $P \cup X$ is a vertex cover for all edges associated with $S$. Obviously,

$$
w\left(V C\left(S, O P T_{S}^{<j}\right)\right) \leq \min _{X} w(X)+\sum_{S^{\prime} \prec S} w\left(V C\left(S^{\prime}, P^{\prime}\right)\right),
$$

where the minimum is taken over all $X$ (a subset of active disks for $S$ with level $j$ ) such that $R-X \cup O P T_{S}^{<j}$ is an independent set, and $P^{\prime}$ is all disks in $X \cup O P T_{S}^{<j}$ that are active for $S^{\prime}$. Remember that here $R$ is the set of all active disks with level at most $j$. Here, $O P T_{S}^{<j}$ denotes the set of all disks in $O P T$ that have level $<j$ and are active for $S$; $S^{\prime} \prec S$ means that $S^{\prime}$ is a child of $S$. We say a relevant $j^{\prime}$-square $S^{\prime \prime} \sqsubseteq S$ if $S^{\prime \prime}=S$ or there exists a relevant square $S^{\prime} \prec S$ and $S^{\prime \prime} \sqsubseteq S^{\prime}$. As did in [3], we will prove

$$
w\left(V C\left(S, O P T_{S}^{<j}\right)\right) \leq \sum_{S^{\prime} \sqsubseteq S} w\left(O P T_{S^{\prime}}^{=j^{\prime}}\right) .
$$

We prove it by induction on the number of relevant squares that were processed by the algorithm.

$$
\begin{aligned}
& w\left(V C\left(S, O P T_{S}^{<j}\right)\right) \\
\leq & \min _{X} w(X)+\sum_{S^{\prime} \prec S} w\left(V C\left(S^{\prime}, P^{\prime}\right)\right) \\
\leq & w\left(O P T_{S}^{=j}\right)+\sum_{S^{\prime} \prec S} w\left(V C\left(S^{\prime}, O P T_{S^{\prime}}^{\leq j}\right)\right) \\
\leq & w\left(O P T_{S}^{=j}\right)+\sum_{S^{\prime} \prec S} \sum_{S^{\prime \prime} \sqsubseteq S^{\prime}} w\left(O P T_{S^{\prime \prime}}^{=j^{\prime \prime}}\right) \\
= & \sum_{S^{\prime} \sqsubseteq S} w\left(O P T_{S^{\prime}}^{=j^{\prime}}\right) .
\end{aligned}
$$

The second inequality is from taking $X=O P T_{S}^{=j}$. Thus, $P^{\prime}$ is all disks in $O P T$ that are active for $S^{\prime}$ with level less than $j^{\prime}$. Notice that, for any child $S^{\prime}$ of $S, O P T_{S^{\prime}}^{<j}=O P T_{S^{\prime}}^{<j^{\prime}}$. The third inequality is from induction. Therefore,

$$
\begin{aligned}
w(A) & =\sum_{S \in \mathcal{R}_{0}} w(V C(S, \emptyset)) \\
& \leq \sum_{S \in \mathcal{R}} w\left(O P T_{S}^{=j}\right) \\
& \leq\left(1+\frac{6}{k}\right) w(O P T) .
\end{aligned}
$$

This finishes the proof of the theorem.

In summary, we obtain a PTAS for the MWVC in all various intersection graphs introduced in this paper.

\section{GRAPH COLORING}

In this section, we study how to color the graphs introduced in this paper. Graf et al. [5] discussed four classes of disk graphs and studied the relations between their chromatic number and the clique number. They proved that, for all such four classes of graphs, their chromatic number is within a constant factor of the clique number. They considered the unit disk graphs, intersection disk graphs (corresponding to disk graph models here), containment disk graphs (corresponding to conflict graph models here), and double disk graphs. Here double disk graph is defined over a set of nodes. Each node $u$ defines two disks $D\left(u, r_{u}\right)$ and $D\left(u, R_{u}\right)$. The double disk graph has an edge $u v$ iff $D\left(u, r_{u}\right)$ intersects $D\left(v, R_{v}\right)$ or $D\left(v, r_{v}\right)$ intersects $D\left(u, R_{u}\right)$.

Let $\delta(G)$ denote the largest $d$ such that $G$ contains a subgraph $H$ in which each vertex has degree at least $d$. It was proved by Szekeres and Wiff [13] that, every graph $G$ can be colored in $\delta(G)+1$ colors. Then Hochbaum [6] presented a method to find the value of $\delta(G)$ and gave an efficient method to color $G$ using $\delta(G)+1$ colors with only $O(|V|+|E|)$ time. For the completeness of presentation, we review the algorithm here. To evaluate $\delta(G)$, it dismantles $G$ by successive removals of vertices of minimum degree and all incident edges. Let $v_{i}$ denote the $i$ th vertex removed from $G$ and $G_{i+1}$ be the graph after $v_{i}$ is removed (set $G_{1}=G$ ). The degree of $v_{i}$ in graph $G_{i}$ is called its valid degree. Set $\delta(G)$ as the maximum valid degree of all nodes. Let $v_{j}$ be the node with the maximum valid degree $\delta(G)$. Then, $v_{j}$ has $\delta(G)$ neighbors among the vertices $v_{j+1}, v_{j+2}, \cdots, v_{n}$. In, addition, all nodes $v_{i}$ with $i>j$ have degree at least $\delta(G)$ in graph $G_{j}$. To color $G$ in no more than $\delta(G)+1$ colors, it scans the sequences of $v_{i}$ 's from $v_{n}$ to $v_{1}$ and assigns to each $v_{i}$ the smallest positive integer not yet assigned to any of its neighbors. Marathe et al. [10] also used this method to color the disk graphs and show an approximation factor of 6 .

THEOREM 8. The above coloring method achieves a constant approximation ratio for all graph models introduced in this paper.

Proof. It was already known that, for any graph $G$, $G$ can be colored by $\delta(G)+1$ colors. Let $H$ be a subgraph such that all nodes have at least $\delta(G)$ degree in $H$. Let $u$ be the node of $H$ with the smallest radius. Let $N_{H}(u)$ be all neighbors of $u$ in $H$. Then $|N(H)| \geq \delta(G)$. 
Consider the induced coloring on $N(u)$ by any coloring of $G$. The node in $N(u)$ with the same color form an independent set. It was proved in Theorem 1 and Theorem 2 that, if $u$ has a radius less than all its neighbors $N_{H}(u)$, then the maximum independent set in $N_{H}(u)$ has size at most 5, 5, 5 and 40 for the disk graph model, ackcommunication graph model, conflict-graph model, and the interference graph model respectively. Let $O P T$ be the chromatic number of the corresponding graph. There are only $O P T-1$ colors for nodes in $N(H)$. Then $|N(H)| \leq$ $5(O P T-1)$ for disk-graph, ack-communication graph and the conflict-graph, while $|N(H)| \leq 40(O P T-1)$ for the interference graph. Thus, the colors used by the above method is at most $5 \cdot O P T-4$ for disk-graph, ack-communication graph and the conflict-graph, while at most $40 \cdot O P T-39$ for the interference graph.

\section{SUMMARY AND FUTURE WORK}

In this paper, we present efficient algorithms and PTASs to approximate MIS, MVC and MGC problems when the wireless networks are modeled by disk graphs, interference graphs, ack-communication graphs, and conflict graphs. We show that MIS and MGC problems can be approximated within 5 or 40 for all graph models presented in this paper by simple methods. We also presents two PTASs for the weighted versions of MIS and MVC for all graph models.

One of the most challenging problems is to design a PTAS for dominating set problem and connected dominating set problem, if it is possible, when the wireless networks are modeled by some graph models introduced in this paper. This has been studied for unit disk graph, however, little is known for the graph models presented in this paper. We leave these problems as future work.

\section{REFERENCES}

[1] Breu, H., And Kirkpatrick, D. G. Unit disk graph recognition is NP-hard. Computational Geometry. Theory and Applications 9, 1-2 (1998), 3-24.

[2] Clark, B., Colbourn, C., and Johnson, D. Unit disk graphs. Discrete Mathematics 86 (1990), 165-177.

[3] Erlebach, T., Jansen, K., and Seidel, E. Polynomial-time approximation schemes for geometric graphs. In Twelfth Annual Symposium on Discrete algorithms (2001).

[4] Golumbic, M. C. Algorithmic Graph Theory and Perfect Graphs. Academic Press, New York., 1980.

[5] Graf, A., Stumpf, M., and Weisenfels, G. On coloring unit disk graphs. Algorithmica 20, 3 (1998), 277-293.

[6] Hochbaum, D. S. Efficient bounds for the stable set, vertex cover, and set packing problems. Discrete Applied Mathematics 6 (1983), 243-254.

[7] Hochbaum, D. S., And MaAss, W. Approximation schemes for covering and packing problems in image processing and vlsi. Journal of ACM 32 (1985), 130-136.

[8] Hunt, H., Marathe, M. V., Radhakrishnan, V., Ravi, S., Rosenkrantz, D. J., and Stearns, R. E. Nc-approximation schemes for np and pspace hard problems for geometric graphs. Journal of Algorithms 26 (1998), 238-274.

[9] Keil, J. M., And Schaefer, D. An optimal algorithm for finding dominating cycles in circular-arc graphs. Discrete Applied Mathematics (1992), 25-34.

[10] Marathe, M. V., Breu, H., Hunt III, H. B., Ravi, S. S., And Rosenkrantz, D. J. Simple heuristics for unit disk graphs. Networks 25 (1995), 59-68.
[11] McKee, T. A., And McMorris, F. R. Topics in Intersection Graph Theory. Vol. 2 of Monographs on Discrete Mathematics and Applications, SIAM, 1999.

[12] RoBerts, F. Graph Theory and Its Applications to Problems of Society. SIAM Press, Philadelphia, PA., 1978.

[13] Szekeres, G., AND Wilf, H. An inequality for the chromatic number of a graph. Journal of Combinatorial Theory 4 (1968), 1-3.

[14] William, T. P. Three-dimensional geometry and topology. The Geometry Center, University of Minnesota, Minneapolis, MN., 1991.

Table 1: Notations used in this paper.

$\mathcal{D} \mid$ a set of 2-dimensional disks

$D_{i} \quad$ a disk with index $i$

$l\left(D_{i}\right) \quad$ the level of a disk $D_{i}$

$v_{i}$ the center of a disk $D_{i}$

$r_{i}$ the transmission radius of a disk $D_{i}$

$k$ parameter to control the quality of solution

$\ell+1$ the total levels of disks

$\mathcal{D}(r, s) \quad$ disks that are active for shifting $(r, s)$

$M W I S(S, I) \quad$ the maximum weighted set of independent disks inside a square $S$ and are $i n$ dependent with disks in $I$

$V C(S, P)$ the minimum weighted set of disks such that $V C(S, P) \cup P$ covers all disks (with level at least $j$ ) inside a $j$-square $S$

$U_{S}$ the set of disks in $U$ that are active for a $j$-square $S$

$U_{S}^{<j} \quad$ the set of disks in $U$ with level less than $j$ that are active for $S$. Similarly we define $U_{\bar{S}}^{\leq j}, U_{S}^{=j}, U_{S}^{>j}$, and $U_{\bar{S}}^{\geq j}$.

$R$ the set of active disks with level at most $j$, intersecting a $j$-square $S$

$J$ a subset of independent active disks with level at most $j$, intersecting a $j$ square $S$

$I$ the set of independent active disks in $J$ with level less than $j$ that intersect a $j$-square $S$

$Q$ the set of active disks not in $J$, with level at most $j$, intersecting a $j$-square $S$. So $Q=R-J$.

$P$ the set of active disks not in $J$, with level less than $j$, intersecting a $j$-square

$C, C_{2}$ upper-bounds on the number of independent active disks with level at most $j$ that intersect a $j$-square 